\title{
Welcoming This Special Issue
}

With the enlightening preface of the guest editors of this very timely STSIP issue on "Modulation Spaces and time-Frequency Analysis", we have not much to add but to express our sincere thanks and appreciation to our colleagues, Professors Karlheinz Gröchenig and Christopher Heil.

Moreover, we are fortunate to have Professor Hans Feichtinger, the originator of this research since 1979 and 1983, to contribute his historical account of this vital area of research. If we are to quote from the preface: "In an average sense, this special issue may be regarded as a celebration of the 25 th birthday of modulation spaces."

Abdul J. Jerri

Founding Executive Editor 